\title{
Probabilistic Graphical Models for the Diagnosis of Analog Electrical Circuits
}

\author{
Christian Borgelt and Rudolf Kruse \\ School of Computer Science, University of Magdeburg \\ Universitätsplatz 2, D-39106 Magdeburg, Germany \\ e-mail: \{borgelt,kruse\}@iws.cs.uni-magdeburg.de
}

\begin{abstract}
We describe an algorithm to build a graphical model-more precisely: a join tree representation of a Markov network-for a steady state analog electrical circuit. This model can be used to do probabilistic diagnosis based on manufacturer supplied information about nominal values of electrical components and their tolerances as well as measurements made on the circuit. Faulty components can be identified by looking for high probabilities for values of characteristic magnitudes that deviate from the nominal values.
\end{abstract}

\section{Introduction}

In electrical engineering several approaches to the diagnosis of electrical circuits have been developed $[10,11]$. Examples are: the fault dictionary approach, which collects a set of common or relevant faults and associates them with (sets of) measurements by which they can be identified [2], the model-based diagnosis of digital circuits based on constraint propagation and an assumption-based truth maintenance system (ATMS) [8], and the simulation of a circuit for different predefined faults to generate training data for a classifier, for example, an artificial neural network $[1,13]$. In particular the diagnosis of digital electrical circuits is well-developed. However, this theory is difficult to transfer to analog circuits due to problems like soft faults (i.e. significant deviations from nominal values) and the non-directional behavior of analog circuits.

The existing methods for the diagnosis of analog circuits suffer from several drawbacks, like difficulties to take tolerances of components and measurements into account. In addition, there is often the need for a predefined set of faults, which are common or relevant for the circuit. In this paper we develop a method that is based on a probabilistic description of the state of the circuit with the help of a graphical model, an approach that is able to handle these problems.

This paper is organized as follows: first we review very briefly in Section 2 the ideas underlying graphical models and in Section 3 the basics of iterative proportional fitting, which we need for initialization purposes. Section 4 discusses some core problems of modeling analog electrical networks in order to justify our approach. In Section 5 we describe our algorithm, which is based on the direct construction of a join tree, and illustrate it with a simple example in Section 6 . Finally, in Section 7, we draw conclusions and point out future work. 


\section{Graphical Models}

In the last decade graphical models have become one of the most popular tools to structure uncertain knowledge about complex domains $[14,9,3]$ in order to make reasoning in such domains feasible $[12,6]$. Their most prominent representatives are Bayes networks, which are based on directed graphs and conditional probability distributions, and Markov networks, which are based on undirected graphs and marginal probability distributions or so-called factor potentials.

More formally: let $V=\left\{A_{1}, \ldots, A_{m}\right\}$ be a set of (discrete) random variables. A Bayes network is a directed graph $G=(V, E)$ of these random variables together with a set of conditional probability distributions, one for each variable given its parents in the graph. A Markov network, on the other hand, is an undirected graph $G=(V, E)$ of the random variables together with a set of functions on the spaces spanned by the variables underlying the maximal cliques ${ }^{1}$ of the graph. In both cases the structure of the graph encodes conditional independence statements between (sets of) random variables that hold in the joint probability distribution represented by the graphical model. This encoding is achieved by node separation criteria, with Bayes networks relying on $d$-separation [12] and Markov networks employing $u$-separation [4].

Conditional independence of $X$ and $Y$ given $Z$, written $X \Perp Y \mid Z$, means

$$
p_{X Y \mid Z}(x, y \mid z) \equiv p_{X \mid Z}(x \mid z) \cdot p_{Y \mid Z}(y \mid z),
$$

where $x, y$ and $z$ are value vectors from the spaces spanned by the random variables in $X, Y$, and $Z$, respectively. For both Bayes networks and Markov networks it can be shown [9] that if the graph encodes only correct conditional independences by $d$ - or $u$-separation, respectively, then the joint probability distribution $p_{V}$ factorizes, namely according to

$$
p_{V}(v) \equiv \prod_{i=1}^{m} p_{A_{i} \mid \operatorname{parents}\left(A_{i}\right)}\left(v\left[\left\{A_{i}\right\}\right] \mid v\left[\operatorname{parents}\left(A_{i}\right)\right]\right) .
$$

for Bayes networks and according to

$$
p_{V}(v) \equiv \prod_{C \in \mathcal{C}} \phi_{C}(v[C])
$$

for Markov networks. Here $v$ is a value vector over the variables in $V$ and $v[X]$ denotes the projection of $v$ to the variables in the set $X$. The $p_{A_{i} \mid \operatorname{parents}\left(A_{i}\right)}$ are conditional probability distributions of the different variables $A_{i}$ given their parents in the directed graph $G$. The set $\mathcal{C}$ is the set of all sets $C$ of variables underlying the maximal cliques of the undirected graph $G$ and the $\phi_{C}$ are functions on the spaces spanned by the variables in the sets $C \in \mathcal{C}$. They are called factor potentials $[6]$ and can be defined in different ways from the corresponding marginal probability distributions.

\footnotetext{
${ }^{1}$ A clique is a complete (fully connected) subgraph and it is called maximal if it is not contained in another complete subgraph.
} 
For reasoning purposes a Bayes or Markov network is often preprocessed into a singly connected structure to avoid update anomalies and incorrect results, which we discuss in somewhat more detail below. The preprocessing consists in forming the moral graph (for Bayes networks only) by "marrying" all parents of a variable, triangulating the graph ${ }^{2}$ and turning the resulting hypertree-structured graph into a join tree [6]. In a join tree there is one node for each maximal clique of the graph it is constructed from. In addition, if a variable (node) of the original graph is contained in two nodes of the join tree, it is also contained in all nodes on the path between these nodes in the join tree. A join tree is usually enhanced by so-called node separators on each edge, which contain the intersection of the variables assigned to the connected join tree nodes.

For join trees there exist efficient evidence propagation methods [6] that are based on a message passing scheme, in which the node separators transmit the information between the nodes. In the approach we present below we work directly with join trees and neglect that our model is actually a Markov network.

\section{Iterative Proportional Fitting}

Iterative proportional fitting (IPF) is a well-known method for adapting the marginal distributions of a given joint probability distribution to desired values [14]. It consists in computing the following sequence of probability distributions:

$$
\begin{aligned}
p_{V}^{(0)}(v) & \equiv p_{V}(v) \\
\forall i=1,2, \ldots: p_{V}^{(i)}(v) & \equiv p_{V}^{(i-1)}(v) \frac{p_{A_{j}}^{*}(a)}{p_{A_{j}}^{(i-1)}(a)}
\end{aligned}
$$

where $j$ is the $((i-1) \bmod |J|+1)$-th element of $J$, the index set that indicates the variables for which marginal distributions are given. $p_{A_{i}}^{*}$ is the desired marginal probability distribution on the domain of the variable $A_{j}$ and $p_{A_{j}}^{(i-1)}$ is the corresponding distribution as it can be computed from $p_{V}^{(i-1)}$ by summing over the values of all variables in $V$ except $A_{j}$.

In each step the probability distribution is modified in such a way that it satisfies one given marginal distribution (namely the distribution $p_{A_{j}}^{*}$ ). However, this will, in general, disturb the marginal for a variable $A_{k}$, which has been processed in a preceding step. Therefore the adaptation has to be iterated, traversing the set of variables several times.

It can be shown that if there is a solution, iterative proportional fitting converges to a (uniquely determined) probability distribution that has the desired marginals as well as some other convenient properties $[5,7]$. Convergence may be checked in practice, for instance, by determining the maximal change of a marginal probability: if this maximal change falls below a user-defined threshold, the iteration is terminated.

\footnotetext{
${ }^{2}$ An undirected graph is called triangulated or chordal if all cycles of length greater than three have a chord, i.e., an edge between two nodes that are nonadjacent in the cycle.
} 

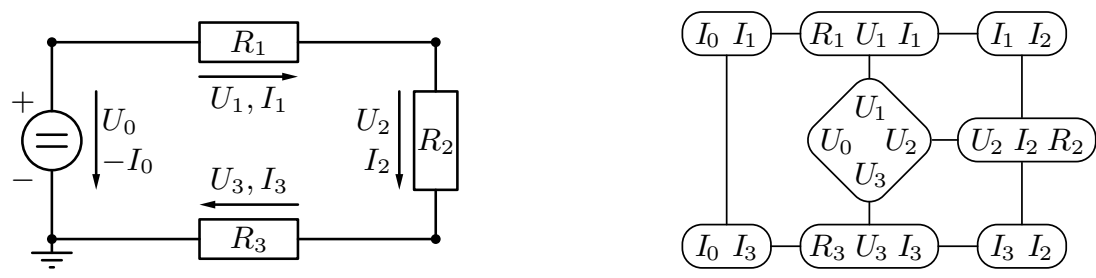

Fig. 1. A simple resistive circuit and an intuitive graph structure for this circuit.

Iterative proportional fitting can easily be extended to probability distributions represented by Markov networks or the corresponding join trees [7]. The idea of this extension is to assign each variable, the marginal distribution of which is to be set, to a maximal clique of the Markov network (or to a node of the join tree it has been turned into), to use steps of iterative proportional fitting to adapt the marginal distributions on the maximal cliques, and to distribute the information added by such an adaptation to the other maximal cliques by join tree propagation.

\section{Modeling Electrical Networks}

In this section we discuss some problems of modeling analog electrical circuits with probabilistic graphical models. Straightforward intuitive approaches fail due to two reasons: (1) cycles in underlying graph structure and (2) difficulties to specify the probability distributions in a plausible and consistent way.

We illustrate these problems with the very simple resistive direct current circuit shown in Figure 1 (left). A very natural approach to construct a graphical model for this circuit would be to set up a clique graph like the one shown in Figure 1 (right), in which there is one node for each electrical law needed to describe the circuit. The nodes at the four corners encode Kirchhoff's junction law for the four corners of the circuit and the diamond-shaped node in the middle represents Kirchoff's mesh law. The remaining three nodes describe the three resistors with Ohm's law. (The two nodes on the left may be removed, since it is $I_{0}=I_{1}=I_{2}=I_{3}$ and thus the two corner nodes on the right suffice.)

The obvious problem with this clique graph is that it is cyclic and thus evidence propagation can lead to inconsistent results. The crucial point is that all four currents must be equal and thus, depending on the resistors, only certain combinations of values for the voltages $U_{1}, U_{2}$ and $U_{3}$ are possible. However, these relations are not enforced by the network, so that actually impossible states of the circuit are not ruled out.

This problem is best understood if we consider a minimum example with binary variables, as shown in Figure 2. It is $\operatorname{dom}(A)=\ldots=\operatorname{dom}(F)=\{0,1\}$. The marginal probability distributions for the four nodes, with $p_{A B C} \equiv p_{F D E}$ and $p_{B D} \equiv p_{C E}$, are shown in Table 1 . Suppose now that $A=1$ is observed. Since 


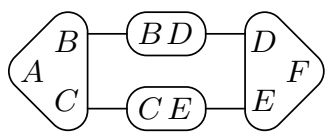

\begin{tabular}{l|ccc|cc|}
\cline { 2 - 5 }$A / F$ & \multicolumn{2}{|c|}{0} & \multicolumn{2}{c|}{1} \\
$B / D$ & 0 & 1 & 0 & 1 \\
\cline { 2 - 6 }$C / E$ & 0 & 0 & 0.25 & 0.25 & 0 \\
& 1 & 0.25 & 0 & 0 & 0.25 \\
\cline { 2 - 5 } & & & &
\end{tabular}

\begin{tabular}{l|c|c|}
\cline { 2 - 3 }$B / C$ & 0 & 1 \\
\cline { 2 - 3 }$D$ & 0.5 & 0 \\
1 & 0 & 0.5 \\
\hline
\end{tabular}

Fig. 2. An illustration of the propagation problem.

Table 1. Probability distributions for the graph structure shown in Figure 2.

this enforces $B=C$ and thus $D=E$, we should get the result $P(F=0)=0$ and $P(F=1)=1$. However, the marginal distributions on the individual variables $B$ and $C$ do not change due to this observation (it is still $P(B=0)=P(B=1)=$ $P(C=0)=P(C=1)=0.5)$. Thus no information is transmitted to the right half of the network, leading to $P(F=0)=P(F=1)=0.5$.

Basically the same problem we encounter for the electrical circuit in the graph structure shown in Figure 1. For instance, if we set the variables for the three resistors to the same value, all voltages $U_{1}, U_{2}$ and $U_{3}$ must be equal. However, this information does not or not completely reach the center node. To cope with this problem we would have to merge nodes in order to obtain an acyclic structure, which, if done inappropriately, can lead to fairly large cliques (and doing it optimally is a non-trivial issue - actually it is NP-hard, since finding an optimal triangulation of an undirected graph is NP-hard [4])

The second problem we encounter when we try to construct a graphical model results from the fact that the electrical laws and the prior information about marginal probability distributions over, for example, the resistor values do not allow for a direct initialization of the quantitative part of the network. In a way, we have too little information. To see this, consider how one may try to build a Bayes network for the circuit shown in Figure 1. We would like to have parentless nodes only for those variables, for which we can specify marginal distributions, that is, for the resistor values and maybe the supply voltage. Every other variable should be the child of one or more variables, with the conditional probability distribution encoding the electrical law that governs the dependence, because we cannot easily specify a marginal distribution for it.

However, this is not possible as Figure 3 demonstrates (note that the current $I_{0}$ is left out, because it must be identical to all other currents anyway). The Bayes network shown in this figure is constructed as follows: First we choose one of the voltages $U_{1}, U_{2}$ and $U_{3}$ as the child for Kirchhoff's mesh law. For reasons of symmetry we choose $U_{2}$, which leads to the edges marked with an $m$, but other choices lead to the same problem in the end. As $U_{1}$ and $U_{3}$ cannot be left parentless, because we cannot easily specify marginal distributions for them, we use Ohm's law to make them dependent on the corresponding currents and resistor values. This leads to the edges marked with an $o$ in the top and bottom row of the network. For the second resistor, however, we make $I_{2}$ the child, 


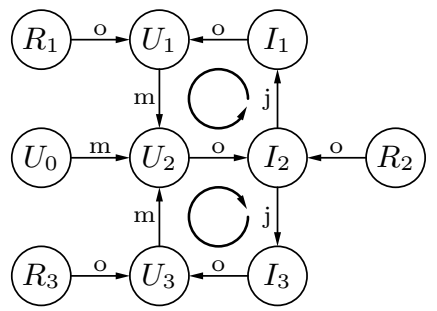

Fig. 3. Attempt at building a Bayes network for the circuit shown in Figure 1: Two cycles result.

because $U_{2}$ already has all the parents it needs and $R_{2}$ should be parentless. This leads to the remaining two edges marked with an $o$. Finally we make $I_{1}$ and $I_{3}$ children of some other variable, because we cannot specify marginal distributions for them easily. The only law left for this is Kirchhoff's junction law, which leads to the edges marked with a $j$. However, the final graph has two cycles and thus cannot be used as a Bayes network.

\section{Constructing the Graphical Model}

In this section we do not use voltages over electrical components anymore, as in Figure 1, but turn to node voltages (potentials). This has advantages not only w.r.t. the measurement process (since node voltages against ground are simpler to measure), but also has some advantages w.r.t. the construction of the graphical model. Note, however, that the problems pointed out in the preceding section are not solved by this transition. In the preceding section we used voltages over components, because this made it easier to demonstrate the core problems.

In the following we describe an algorithm to construct a join tree representation of a Markov network for an analog electrical circuit. Let a time-invariant $n+1$ node, $b$ branch steady state circuit with known topology be given, the nodes of which are accessible terminals for measurements. One of them is taken as a reference (ground) and the node voltages are used to study the circuit. We assume that for each component the electrical law that governs its behavior (for example, Ohm's law for a resistor), its nominal value(s) and a tolerance provided by the manufacturer are known. We use the following notation:

$$
\begin{aligned}
& U_{i}, i=0, \ldots, n-1: \text { node voltages } \\
& I_{j}, \quad j=0, \ldots, b-1: \text { branch currents } \\
& R_{k}, k=0, \ldots, b-1: \text { branch resistances. }
\end{aligned}
$$

(Note that all magnitudes may be complex numbers, making it possible to handle steady state alternating current circuits. For reasons of simplicity, however, we confine ourselves to direct current circuits here.)

To build a join tree of a Markov network for this circuit, we have to find partitions of the set of variables $V=\left\{U_{0}, \ldots, U_{n-1}, I_{0}, \ldots, I_{b-1}, R_{0}, \ldots, R_{b-1}\right\}$ into three disjoint subsets $X_{1}, X_{2}$ and $X_{3}$, such that the variables in $X_{1}$ and $X_{2}$ are conditionally independent given the variables in $X_{3}$. That is, if the values of 
the variables in $X_{3}$ are fixed, a change of the value of a variable in $X_{1}$ has no effect on the values of the variables in $X_{2}$ and vice versa.

To find such partitions, we consider virtual cross-sections through the circuit (only through wires, not through components). Each of these cross-sections defines a set of variables, namely the voltages of the wires that are cut and the currents flowing through them. Since this set of variables obviously has the property of making the variables on one side of the cross-section independent of those on the other side (and thus satisfies the conditional independence property), we call it a separator set. We select a set of cross-sections so that each component is enclosed by two or more cuts or is cut off from the rest of the circuit by a single cut (terminal cross-section). Then the electrical law governing a component describes how the variables of its enclosing cross-sections relate to each other. Note that there are usually several ways of selecting the cross-sections and that an appropriate selection is crucial to the complexity of the network. However, selecting appropriate cross-sections is easier than finding good node mergers in the approach discussed above.

Given a set of cross-sections we construct the join tree as follows: the separator sets form the node separators. For each circuit part (containing one component) we create a node containing the union of the separator sets of the bounding cross-sections. In addition, we create a node for each component, comprising the variables needed to describe its behavior, and connect it to the node corresponding to the circuit part the component is in. If the component node contains currents not yet present in the circuit part node, we add these currents to it. The connection is made through an appropriate node separator, containing the intersection of the sets of variables assigned to the connected nodes.

Next this initial graphical model is simplified in two steps. In the first step, the number of variables is reduced by exploiting trivial Kirchhoff junction equations (like the identity of two currents). In the second step, we merge adjacent nodes where the variables in one of them is a subset of the variables in the other. The result is the qualitative part of the graphical model, i.e. the graph structure of the join tree, enhanced with node separators.

To find the quantitative part (the probability distributions), we initialize all node distributions to uniform. Next we enforce the component laws as well as Kirchhoff's laws (wherever applicable) by zeroing the entries of the probability distributions that correspond to impossible value combinations. Finally we incorporate the manufacturer supplied information about nominal values and tolerances by iterative proportional fitting (see Section 3), thus setting the marginal component distributions. The resulting graphical model can then be used to diagnose the modeled circuit by propagating node voltage measurements.

From the theory of evidence propagation in graphical models and in particular in join trees it is well known that the computational complexity of operations (iterative proportional fitting and evidence propagation) is governed by the size of the node distributions, which depends on the number of variables in a join tree node and the sizes of their domains. If the distributions can be kept small by a proper selection of cross-sections, the computation is very efficient. 

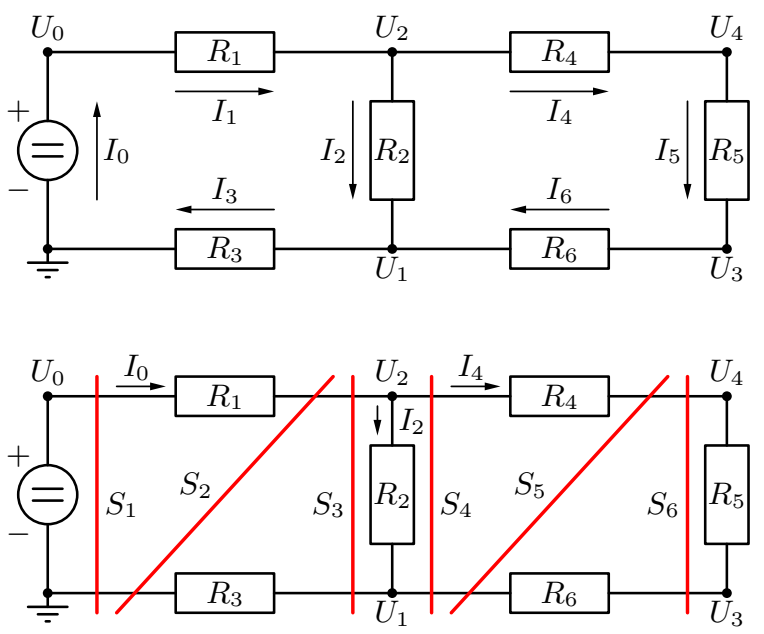

Fig. 4. Another very simple resistive circuit.

Fig. 5. The resistive circuit with cross-sections.

\section{A Simple Example}

To illustrate our approach we consider the simple resistive circuit shown in Figure 4 , where $n=5, b=7$. It is fed by a voltage supply $U_{0}$, whose internal resistance $R_{0}$ we assume to be zero. The set of (real valued) variables is $V=\left\{U_{0}, \ldots, U_{4}, I_{0}, \ldots, I_{6}, R_{0}, \ldots, R_{6}\right\}$. We select the set of six cross-sections $S_{1}$ to $S_{6}$ that are shown in Figure 5. As an example of the conditional independences consider the cross-section $S_{3}$ : once we know the voltage of the cut wires $\left(U_{1}\right.$ and $\left.U_{2}\right)$ and the currents through them $\left(I_{1}\right.$ and $\left.I_{3}, I_{3}=I_{1}\right)$, all the magnitudes to the left of $S_{3}$ become independent of those to the right of $S_{3}$.

The initial graphical model, as it is constructed from the separator sets, is shown in Figure 6 . The node separators (rectangles) are labeled by the crosssections $S_{1}$ to $S_{6}$ they correspond to. The nodes are drawn with rounded corners and thicker lines. To simplify the network, we exploit $I_{0}=I_{1}=I_{3}$ and $I_{4}=$ $I_{5}=I_{6}$. Furthermore, we merge (1) the four leftmost nodes (two from the top row and two from the bottom row), (2) the third and the fourth node on the top row and (3) the two rightmost nodes (the last nodes from the top and the bottom row). The result is shown in Figure 7 .

For our experiments we implemented the described method for this example and a discrete Markov network in C. ${ }^{3}$ We discretized the continuous ranges of values as follows ${ }^{4}$ : resistors: 1 to $5 \Omega$ with $1 \Omega$ steps, voltages: 0 to $20 \mathrm{~V}$ with $1 \mathrm{~V}$ steps, currents: 0 to $4 \mathrm{~A}$ with $1 \mathrm{~A}$ steps. For the six resistors we set an initial

${ }^{3}$ We plan to make the $\mathrm{C}$ sources available at the URL http://fuzzy.cs.unimagdeburg.de/ borgelt/software.html

${ }^{4}$ An alternative to handle metric attributes, which comes to mind immediately, is a Gaussian network. Unfortunately, in its standard form (that is, with a covariance matrix) a Gaussian network is restricted to linear dependences. Ohm's law, however, specifies a nonlinear dependences as it involves the product of two quantities. 

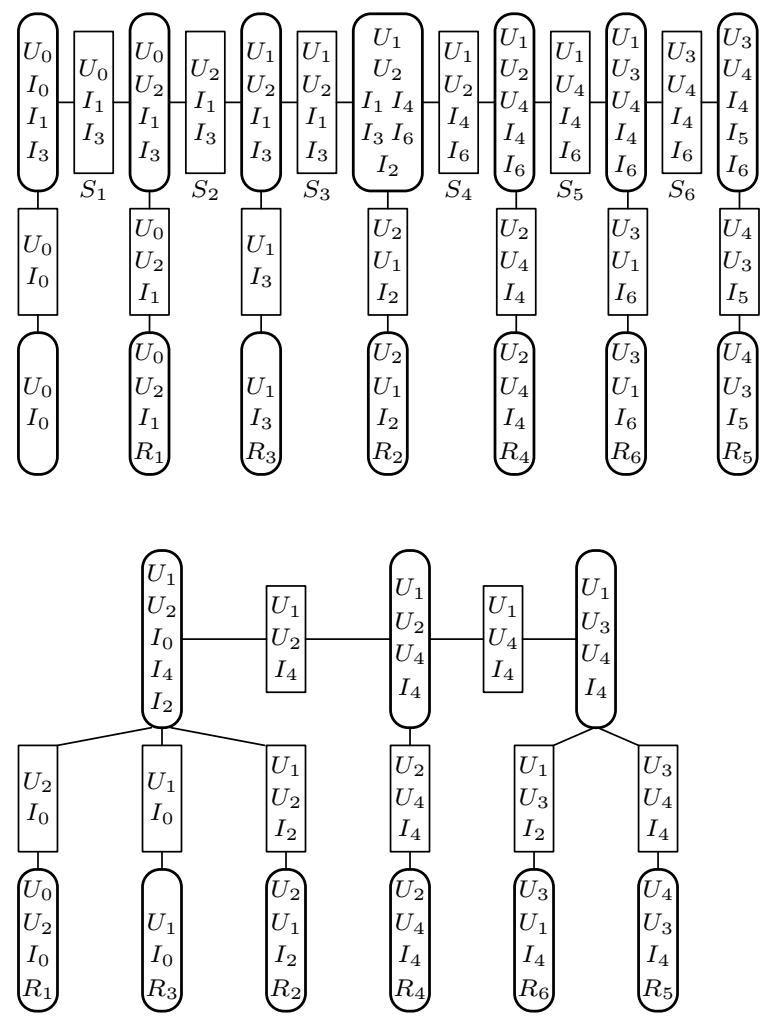

Fig. 6. Initial graphical model for the example.
Fig. 7. Simplified graphical model for the example.

probability distribution that is roughly normal and centered at $3 \Omega$, that is, for $i=1, \ldots, 6: p_{R_{i}}(r)=(0.1,0.2,0.4,0.2,0.1)$.

The initial probability distributions are determined as described in Section 5 , that is, by enforcing the electrical laws and incorporating the resistor maginals by iterative proportional fitting. To mitigate the effects of the discretization of the value ranges, we set a zero probability only if there is no combination of values from the represented intervals that is valid, i.e., satisfies the electrical law. With a threshold of $10^{-6}$ the iterative proportional fitting procedure converges after 5 iterations. This yields the diagnostic network.

Next we set the voltage supply to $20 \mathrm{~V}$ and propagate this information using join tree propagation. This changes the marginals of the resistors only slightly as can be seen on the left in Table 2. Suppose now that we measure the node voltage $U_{4}$ and find it to be $5 \mathrm{~V}$. Propagating this evidence yields the resistor marginals shown on the right in Table 2. It can be seen that due to the measurement the distributions for $R_{1}$ and $R_{3}$ change considerably, indicating that at least resistor $R_{3}$ is highly likely to deviate from its nominal value. 


\begin{tabular}{|l|ccccc|ccccc|}
\hline & \multicolumn{6}{|c|}{$U_{0}=20$} & \multicolumn{4}{c|}{$U_{0}=20 \wedge U_{4}=5$} \\
\hline$R_{1}$ & .11 & .22 & .39 & .19 & .09 & .00 & .04 & .33 & .32 & .31 \\
\hline$R_{2}$ & .09 & .18 & .41 & .21 & .11 & .17 & .23 & .38 & .16 & .07 \\
\hline$R_{3}$ & .12 & .22 & .40 & .18 & .08 & .53 & .29 & .15 & .03 & .00 \\
\hline$R_{4}$ & .11 & .21 & .40 & .19 & .09 & .05 & .15 & .39 & .27 & .15 \\
\hline$R_{5}$ & .11 & .21 & .40 & .19 & .09 & .16 & .25 & .37 & .16 & .07 \\
\hline$R_{6}$ & .11 & .21 & .40 & .19 & .09 & .16 & .25 & .37 & .16 & .07 \\
\hline
\end{tabular}

Table 2. Resistor marginals after propagating the supply voltage $U_{0}=20$ and the measurement $U_{4}=5$.

\section{Conclusions and Future Work}

We presented a method for modeling and diagnosing analog electrical circuits that exploits probabilistic information about production tolerances of electrical components. It consists of: the construction of a join tree representation of a Markov network from a set of cross-sections of an analog electrical circuit; the iterative proportional fitting procedure for the initialization of the probability distributions; and the join tree propagation algorithm for the incorporation of measurements. For our experiments we used a simple example to keep things comprehensible, but the approach is fully general and can be applied to any steady state, alternating or direct current electrical circuit. Faults like shortcuts or open connections can easily be included by adding them as possible states to the variable(s) describing a circuit component.

In the future we plan to make our method more efficient by exploiting the sparsity of the (discrete) probability distributions (the electrical laws rule out a large number of value combinations) and by using parameterized continuous distributions. Furthermore, we plan to develop a theory of how to select measurements in a diagnosis process. The basic idea is to propagate possible outcomes of measurements through the network, to compute (and to aggregate) the resulting reductions in entropy of the distributions on component values, and finally to select the measurement that leads to the highest expected entropy reduction (similar to the approach suggested in [8]).

\section{References}

1. F. Aminian, M. Aminian, and H.W. Collins. Analog Fault Diagnosis of Actual Circuits Using Neural Networks. IEEE Trans. Instrumentation and Measurement 51(3):544-550. IEEE Press, Piscataway, NJ, USA 2002

2. J.W. Bandler and A.E. Salama. Fault Diagnosis of Analog Circuits. Proc. IEEE 73:1279-1325. IEEE Press, Piscataway, NJ, USA 1985

3. C. Borgelt and R. Kruse. Graphical Models - Methods for Data Analysis and Mining. J. Wiley \& Sons, Chichester, UK 2002

4. E. Castillo, J.M. Gutierrez, and A.S. Hadi. Expert Systems and Probabilistic Network Models. Springer-Verlag, New York, NY, USA 1997

5. I. Csiszar. I-Divergence Geometry of Probability Distributions and Indirect Observations. Studia Scientiarum Mathematicarum Hungarica 2:299-318. Hungarian Academy of Sciences, Budapest, Hungary 1975 
6. F.V. Jensen. An Introduction to Bayesian Networks. UCL Press, London, UK 1996

7. R. Jiroušek and S. Pøeuèil. On the Effective Implementation of the Iterative Proportional Fitting Procedure. Computational Statistics and Data Analysis 19:177189. Int. Statistical Institute, Voorburg, Netherlands 1995

8. J. de Kleer and B.C. Williams. Diagnosing Multiple Faults. Artificial Intelligence 32(1):97-130. Elsevier Science, New York, NY, USA 1987

9. S.L. Lauritzen. Graphical Models. Oxford University Press, Oxford, UK 1996

10. R.-W. Liu, ed. Selected Papers on Analog Fault Diagnosis. IEEE Press, New York, NY, USA 1987

11. R.-W. Liu. Testing and Diagnosis of Analog Circuits and Systems. Van Nostrand Reinhold, New York, NY, USA 1991

12. J. Pearl. Probabilistic Reasoning in Intelligent Systems: Networks of Plausible Inference (2nd edition). Morgan Kaufman, San Mateo, CA, USA 1992

13. R. Spina and S. Upadhyaya. Linear Circuit Fault Diagnosis Using Neuromorphic Analyzers. IEEE Trans. Circuits and Systems II 44(3):188-196. IEEE Press, Piscataway, NJ, USA 1997

14. J. Whittaker. Graphical Models in Applied Multivariate Statistics. J. Wiley \& Sons, Chichester, UK 1990 